\title{
Applications of $\delta$-function perturbation to the pricing of derivative securities
}

\author{
Marc Decamps ${ }^{\mathrm{a}, *}$ Ann De Schepper ${ }^{\mathrm{b}}$ Marc Goovaerts ${ }^{\mathrm{a}, \mathrm{c}}$ \\ ${ }^{a}$ K.U.Leuven, FETEW, Naamsestraat 69, 3000 Leuven, Belgium \\ ${ }^{\mathrm{b}}$ University of Antwerp, Faculty of Applied Economics, Prinsstraat 13, 2000 \\ Antwerp, Belgium \\ ${ }^{\mathrm{c}}$ University of Amsterdam, Roetersstraat 11, 1018 WB Amsterdam, Netherlands
}

\begin{abstract}
In the recent econophysics literature, the use of functional integrals is widespread for the calculation of option prices. In this paper, we extend this approach in several directions by means of $\delta$-function perturbations. First, we show that results about infinitely repulsive $\delta$-function are applicable to the pricing of barrier options. We also introduce functional integrals over skew paths that give rise to a new European option formula when combined with $\delta$-function potential. We propose accurate closed-form approximations based on the theory of comonotonic risks in case the functional integrals are not analytically computable.
\end{abstract}

Key words: functional integrals, local time, comonotonicity, skew Brownian motion, option pricing, $\delta$-function perturbation PACS: 02.30.Mv, 02.50.Ey, 05.40.+j, 31.15.K

\footnotetext{
$\bar{*}$ Corresponding author.

Email addresses: marc.decamps@econ.kuleuven.ac.be (Marc Decamps), ann.deschepper@ua.ac.be (Ann De Schepper), marc.goovaerts@econ.kuleuven.ac. be (Marc Goovaerts).
}

Preprint submitted to Physica A 4 January 2005 


\section{Introduction}

Diffusion processes have proven over the years to be very convenient to model the uncertainty in the economy. In particular, stochastic differential equations

$$
d X_{t}=\mu\left(X_{t}\right) d t+\sigma\left(X_{t}\right) d W_{t}
$$

where $\left\{W_{t}, t \geq 0\right\}$ is a standard Brownian motion, are popular in finance to capture the dynamic of various risky assets including stocks, interest rates, volatilities, etc. The function $\mu(x)$ and $\sigma(x)^{2}$ are called the instantaneous drift and diffusion coefficients and the operator

$$
G=\frac{1}{2} \sigma(x)^{2} \frac{d^{2}}{d x^{2}}+\mu(x) \frac{d}{d x}
$$

is the associated generator. Next to the issue of determining parsimonious models for risky assets, the pricing of securities against undesired fluctations of the economy has received considerable attention. A security (or contingent claim) is a financial contract whose value depends on a more basic asset. Call options, bonds, caplets, floorlets, swaps, etc are all famous examples of contingent claims. The volumes of traded securities are in general much higher than those of the underlying assets, and economical agents expect from models to provide fair prices of securities. Path-dependent exotic derivatives have also become popular in the over-the-counter (OTC) market in the last decades. Examples of exotic derivatives are lookback options and barrier options.

As we show in this paper, it might be necessary for financial applications to impose an impermeable (or even a permeable) barrier at some level $a$ and to specify the behaviour of the diffusion when hitting the barrier. In case an absorbing boundary is imposed, the diffusion equation (1) is not altered until the first time the process hits the barrier. Now, if we impose a reflecting boundary at a level $a$, this generates an additional term in the drift, proportional to a Dirac $\delta$-function. Stochastic processes whose drift is a generalized function, are solutions of diffusion equations involving their (symmetric) local time, see e.g. Le Gall [17]. The symmetric local time of the process $X=\left\{X_{t}, t \geq 0\right\}$

measures the time spent in the vicinity of the point $a$, and it can be expressed as

$$
L_{t}^{a}(X)=\frac{1}{2}\left(L_{t}^{a-}(X)+L_{t}^{a+}(X)\right)
$$

where the left-local time $L_{t}^{a-}$ is defined by

$$
L_{t}^{a-}(X)=\lim _{\epsilon \rightarrow 0} \frac{1}{\epsilon} \int_{0}^{t} 1_{] a-\epsilon, a]}\left(X_{s}\right) d\langle X, X\rangle_{s}
$$


and the right-local time $L_{t}^{a+}$ by

$$
L_{t}^{a+}(X)=\lim _{\epsilon \rightarrow 0} \frac{1}{\epsilon} \int_{0}^{t} 1_{[a, a+\epsilon]}\left(X_{s}\right) d\langle X, X\rangle_{s} .
$$

If left- and right-local time are equal, the local time is continuous in $a$ (in this case, we can bravely think of $L_{t}^{a}(X)$ as $\left.\int_{0}^{t} \delta\left(X_{s}-a\right) d s\right)$. However, if the process is reflected in $a$ (with starting point $x \geq a$ ), the local time is discontinuous in $a$. Indeed, in this case $L_{t}^{a-}(X)=0$ and as a consequence $L_{t}^{a}(X)=\frac{1}{2} L_{t}^{a+}(X)$. Moreover, in the case of a reflecting boundary, equation (1) has to be modified into

$$
d X_{t}=\mu\left(X_{t}\right) d t+\sigma\left(X_{t}\right) d W_{t}+\frac{1}{2} d L_{t}^{a+}(X)
$$

see also Revuz \& Yor [24]. More generally, it can be shown that the solution of the diffusion equation

$$
d X_{t}=\mu\left(X_{t}\right) d t+\sigma\left(X_{t}\right) d W_{t}+(2 \alpha-1) d L_{t}^{a}(X)
$$

for some constant $0 \leq \alpha \leq 1$ behaves like the process $X$ except at the level $a$ where it reflects to above with probability $\alpha$ and to below with probability $1-\alpha$. We can interpret the point $a$ as a permeable barrier where the local time is discontinuous : $\alpha L_{t}^{a-}(X)=(1-\alpha) L_{t}^{a+}(X)$, or equivalently

$$
L_{t}^{a}(X)=\frac{L_{t}^{a-}(X)}{2(1-\alpha)}=\frac{L_{t}^{a+}(X)}{2 \alpha} .
$$

When $\mu(x)=0$ and $\sigma(x)=1$, the solution of $(3)$ is the skew Brownian motion, see e.g. Itô \& McKean [15]. The intriguing properties of the skew Brownian motion have led to applications in various disciplines. We can cite Zhang [20] in theoretical physics or Cantrell \& Cosner [3] in biology. In this paper, we introduce functional integrals over skew Brownian paths to derive a new option formula ${ }^{1}$.

Diffusion processes can be related to quantum mechanical particles with convenient Hamiltonian as explained in detail e.g. in Goovaerts et al. [11]. As a consequence, the transition probability of $X$ describing its time-evolution can be formulated by means of a functional integral. The introduction of a boundary at the level $a$ causes an extra term proportional to a $\delta$-function in the potential. For the absorbing case, we need to add $\gamma L_{t}^{a}(X)$ in the integrand of the Brownian functional integral with $\gamma$ going to infinity, see e.g. Grosche [13,14] and Goovaerts et al. [12]. We can also obtain an expression for the transition probability of reflecting diffusions using functional integrals

$\overline{1}$ In the recent research in the econophysics field, the use of path integrals for the calculation of security and option prices is widespread. Without claiming any exhaustiveness, we refer e.g. to $[2,5,16,18,21-23,25]$. 
over reflecting Brownian motion with the local time in the integrand as defined by Carreau [4]. We rely on results about $\delta$-function perturbation to solve the Green's function of functional integrals involving the local time, see e.g. Goovaerts et al. [12]. To our knowledge, functional integrals over skew Brownian paths have not yet been investigated neither in the mathematical physics nor in the probability literature. In this paper, we fill the gap and we give some applications in econophysics as well as appropriate approximations.

The paper is organized as follows. We start in section 2 with a brief overview of functional integrals and we construct a functional integral with respect to the skew Brownian motion. In section 3, we adapt the results on $\delta$-function perturbation to this particular situation. In section 4 , we derive closed-form approximations for functional integrals with the local time in the integrand based on the theory of comonotonic risks. We propose two applications to the pricing of financial derivatives. In section 5, we price double barrier options using infinitely repulsive $\delta$-function. In section 6 , we obtain a closed-form expression for the price of a European option when the local volatility is discontinuous by means of a skew functional integral. The accuracy of the approximation is illustrated on both examples.

\section{Functional integrals}

We can demonstrate in a straightforward way by means of an expansion that the Brownian functional integral

$$
I_{[V(x)]}\left(x, x_{t}, t\right)=\int_{(0, x)}^{\left(t, x_{t}\right)} D x(s) e^{-\frac{1}{2} \int_{0}^{t} \dot{x}^{2}(s) d s-\int_{0}^{t} V(x(s)) d s}
$$

is the solution of the Schrödinger equation in imaginary time

$$
-\frac{d}{d t} I\left(x, x_{t}, t\right)=\left[-\frac{1}{2} \frac{d^{2}}{d x^{2}}+V(x)\right] I\left(x, x_{t}, t\right)
$$

with condition $I_{[V(x)]}\left(x, x_{t}, 0\right)=\delta\left(x_{t}-x\right)$. The Green's function

$$
G_{[V(x)]}\left(x, x_{t}, s\right)=\int_{0}^{+\infty} e^{-s t} I_{[V(x)]}\left(x, x_{t}, t\right) d t
$$

which is the Laplace transform of the previous functional integral, is the solution of the equation

$$
-s G\left(x, x_{t}, s\right)=\left[-\frac{1}{2} \frac{d^{2}}{d x^{2}}+V(x)\right] G\left(x, x_{t}, s\right)-\delta\left(x_{t}-x\right) .
$$


We now extend the construction of Carreau [4] and we define the skew Brownian functional integral

$$
I_{[V(x)]}^{\alpha}\left(x, x_{t}, t\right)=\int_{(0, x)}^{\left(t, x_{t}\right)} D x_{\alpha}(s) e^{-\frac{1}{2} \int_{0}^{t} \dot{x}_{\alpha}^{2}(s) d s-\int_{0}^{t} V\left(x_{\alpha}(s)\right) d s}
$$

for some constant $0 \leq \alpha \leq 1$. The symbol $D x_{\alpha}(s) e^{-\frac{1}{2} \int_{0}^{t} \dot{x}_{\alpha}^{2}(s) d s}$ has to be interpreted as the skew Brownian measure, for which

$$
\begin{aligned}
\int_{(0, x)}^{\left(t, x_{t}\right)} D x_{\alpha}(s) e^{-\frac{1}{2} \int_{0}^{t} \dot{x}_{\alpha}^{2}(s) d s}= & \frac{1}{\sqrt{2 \pi t}} e^{-\frac{1}{2 t}\left|x_{t}-x\right|^{2}} \\
& +\operatorname{sign}\left(x_{t}-a\right) \frac{(2 \alpha-1)}{\sqrt{2 \pi t}} e^{-\frac{1}{2 t}\left(\left|x_{t}-a\right|+|a-x|\right)^{2}}
\end{aligned}
$$

that gives non-zero weight to continuous paths reflected above with probability $\alpha$ when they hit the level $a$. The skew functional integral (9) is the solution of the Schrödinger equation (6) subject to the boundary condition

$$
(1-\alpha) \frac{d}{d x} I^{\alpha}\left(a-, x_{t}, t\right)=\alpha \frac{d}{d x} I^{\alpha}\left(a+, x_{t}, t\right)
$$

In case $\alpha=0$ or $\alpha=1$, we recover functional integrals over reflecting Brownian paths as introduced by Carreau [4].

\section{$3 \delta$-function perturbation}

The Green's function of the one dimensional $\delta$-potential can be solved by summing all the terms of the perturbation expansion

$$
\begin{aligned}
I_{[V(x)+\gamma \delta(x-a)]}\left(x, x_{t}, t\right)= & \int_{(0, x)}^{\left(t, x_{t}\right)} D x(s) e^{-\frac{1}{2} \int_{0}^{t} \dot{x}^{2}(s) d s-\int_{0}^{t} V(x(s)) d s-\gamma L_{t}^{a}(x)} \\
= & I_{[V(x)]}\left(x, x_{t}, t\right)+\sum_{n=1}^{+\infty} \frac{(-1)^{n}}{n !}\left(\prod_{j=1}^{n} \int_{0}^{t_{j+1}} d t_{j} \int_{-\infty}^{+\infty} d x_{j}\right) \\
& \times I_{[V(x)]}\left(x, x_{1}, t_{1}\right) \gamma \delta\left(x_{1}-a\right) I_{[V(x)]}\left(x_{1}, x_{2}, t_{2}-t_{1}\right) \\
& \times \ldots \times \gamma \delta\left(x_{n}-a\right) I_{[V(x)]}\left(x_{n}, x_{t}, t-t_{n}\right),
\end{aligned}
$$

as done in Goovaerts et al. [12]. The Green's function of the Brownian functional integral $I_{[V(x)+\gamma \delta(x-a)]}$ can be expressed by means of the regular Green's function 


$$
\begin{aligned}
G_{[V(x)+\gamma \delta(x-a)]}\left(x, x_{t}, s\right)= & G_{[V(x)]}\left(x, x_{t}, s\right) \\
& -\frac{G_{[V(x)]}(x, a, s) G_{[V(x)]}\left(a, x_{t}, s\right)}{G_{[V(x)]}(a, a, s)+1 / \gamma} .
\end{aligned}
$$

It is straightforward to check that $\lim _{\gamma \rightarrow+\infty} G_{[V(x)+\gamma \delta(x-a)]}\left(a, x_{t}, s\right)=0$, which corresponds to the Dirichlet solution of the Schrödinger equation (8) denoted by $G_{[V(x)]}^{D}\left(x, x_{t}, t\right)$. For the free particle $(V(x)=0)$, we obtain the absorbing Brownian motion, for which the result is well known :

$$
I_{(0)}^{D}\left(x, x_{t}, t\right)=\frac{1}{\sqrt{2 \pi t}}\left(e^{-\frac{1}{2 t}\left|x_{t}-x\right|^{2}}-e^{-\frac{1}{2 t}\left(\left|x_{t}-a\right|+|a-x|\right)^{2}}\right)
$$

with $x \geq a$ and $x_{t} \geq a$.

For skew functional integrals, $\delta$-function perturbation can be adapted taking into account that $I_{[V(x)]}^{\alpha}\left(x, x_{t}, t\right)$ is discontinous at the level $x_{t}=a$. As a consequence, the functional integral $I_{[V(x)+\delta(x-a+)]}^{\alpha}\left(x, x_{t}, t\right)$ is not the same as the funtional integral $I_{[V(x)+\delta(x-a-)]}^{\alpha}\left(x, x_{t}, t\right)$. The discontinuity of the local time of the skew Brownian motion implies that the functional integral $I_{[V(x)]}^{\alpha, \gamma}\left(x, x_{t}, t\right)=I_{[V(x)+\gamma \delta(x-a)]}^{\alpha}\left(x, x_{t}, t\right)$ satisfies

$$
\begin{aligned}
I_{[V(x)]}^{\alpha, \gamma}\left(x, x_{t}, t\right) & =\int_{(0, x)}^{\left(t, x_{t}\right)} D x_{\alpha}(s) e^{-\frac{1}{2} \int_{0}^{t} \dot{x}_{\alpha}^{2}(s) d s-\int_{0}^{t} V\left(x_{\alpha}(s)\right) d s-\frac{\gamma}{2 \alpha} L_{t}^{a+}\left(x_{\alpha}\right)} \\
& =\int_{(0, x)}^{\left(t, x_{t}\right)} D x_{\alpha}(s) e^{-\frac{1}{2} \int_{0}^{t} \dot{x}_{\alpha}^{2}(s) d s-\int_{0}^{t} V\left(x_{\alpha}(s)\right) d s-\frac{\gamma}{2(1-\alpha)} L_{t}^{a-}\left(x_{\alpha}\right)}
\end{aligned}
$$

With similar computations as in Goovaerts et al. [12], we can check that

$$
\begin{aligned}
G_{[V(x)+\gamma /(2 \alpha) \delta(x-a+)]}^{\alpha}\left(x, x_{t}, s\right)= & G_{[V(x)]}^{\alpha}\left(x, x_{t}, s\right) \\
& -\frac{G_{[V(x)]}^{\alpha}(x, a+, s) G_{[V(x)]}^{\alpha}\left(a+, x_{t}, s\right)}{G_{[V(x)]}^{\alpha}(a+, a+, s)+2 \alpha / \gamma} \\
= & G_{[V(x)]}^{\alpha}\left(x, x_{t}, s\right) \\
& -\frac{G_{[V(x)]}^{\alpha}(x, a-, s) G_{[V(x)]}^{\alpha}\left(a-, x_{t}, s\right)}{G_{[V(x)]}^{\alpha}(a-, a-, s)+2(1-\alpha) / \gamma} \\
= & G_{[V(x)+\gamma /(2-2 \alpha) \delta(x-a-)]}^{\alpha}\left(x, x_{t}, s\right) .
\end{aligned}
$$

The skew functional integral $I_{[V(x)]}^{\alpha, \gamma}\left(x, x_{t}, t\right)$ is hence the solution of the Schrödinger equation (6) subject to the boundary condition

$$
\begin{array}{r}
(1-\alpha)\left(\frac{d}{d x} I^{\alpha, \gamma}\left(a-, x_{t}, t\right)-\gamma I^{\alpha, \gamma}\left(a-, x_{t}, t\right)\right) \\
=\alpha\left(\frac{d}{d x} I^{\alpha, \gamma}\left(a+, x_{t}, t\right)-\gamma I^{\alpha, \gamma}\left(a+, x_{t}, t\right)\right) .
\end{array}
$$


The boundary condition results from the derivation with respect to $x$ of the relations (13) rewritten as

$$
\begin{aligned}
& G_{[V(x)]}^{\alpha, \gamma}\left(x, x_{t}, s\right) \\
& \quad=G_{[V(x)]}^{\alpha}\left(x, x_{t}, s\right)-\frac{\gamma}{2 \alpha} G_{[V(x)]}^{\alpha, \gamma}\left(a+, x_{t}, s\right) G_{[V(x)]}^{\alpha}(x, a+, s) \\
& \quad=G_{[V(x)]}^{\alpha}\left(x, x_{t}, s\right)-\frac{\gamma}{2(1-\alpha)} G_{[V(x)]}^{\alpha, \gamma}\left(a-, x_{t}, s\right) G_{[V(x)]}^{\alpha}(x, a-, s) .
\end{aligned}
$$

\section{Closed-form approximation}

In order to compute the skew functional integral $I_{[V(x)]}^{\alpha}\left(x, x_{t}, t\right)$, one could rely on its spectral representation

$$
I_{[V(x)]}^{\alpha}\left(x, x_{t}, t\right)=\sum_{E} e^{-E t} \Psi_{E}(x) \Psi_{E}\left(x_{t}\right)
$$

where the sum is to be interpreted as an integral when the spectrum is continuous, see e.g. Itô \& McKean [15]. The properly normalized eigenfunctions $\Psi_{E}(x)$ are the continuous solutions of the Sturm-Liouville problem

$$
H \Psi_{E}(x)=E \Psi_{E}(x)
$$

subject to the condition $(1-\alpha) \frac{d}{d x} \Psi_{E}(a-)=\alpha \frac{d}{d x} \Psi_{E}(a+)$ where $H=-\frac{1}{2} \frac{d^{2}}{d x^{2}}+$ $V(x)$ is the Hamiltonian. The Sturm-Liouville problem (16) can be solved as long as analytical solutions exist on the intervals $(-\infty, a]$ and $[a,+\infty)$. Unfortunately, the eigenfunctions expansion (15) converges slowly for short time $t$ which is the case for financial applications. Moreover, for most of the stock price models, the spectrum is continuous and makes the computation of (15) very extensive. In this section, we propose analytical approximations that render skew functional integrals applicable to the pricing of options.

\subsection{Methodology}

In most cases, an exact calculation of the functional integrals is not possible. In this section we will propose a solution appropriate to functional integrals with $\delta$-potential in the integrand. We derive an analytical upper and lower bound for such functional integrals, which can also be combined to give an accurate approximation. Our methodology is based on a decomposition of the function $V$ appearing in the potential of the functional integral $I_{[V(x)]}^{\alpha}\left(x, x_{t}, t\right)$. This decomposition $V(x)=V_{1}(x)+V_{2}(x)$ has to be chosen in such a way that the functional integral corresponding to $V_{2}(x)$ is perfectly tractable. It 
is for the more difficult part denoted by $V_{1}(x)$, that we will work with an approximation.

It can be shown in a straightforward way, that for any decomposition $V(x)=$ $V_{1}(x)+V_{2}(x)$, the functional integral $I_{[V(x)]}^{\alpha}\left(x, x_{t}, t\right)$ can be written as

$$
I_{[V(x)]}^{\alpha}\left(x, x_{t}, t\right)=I_{\left[V_{2}(x)\right]}^{\alpha}\left(x, x_{t}, t\right) E_{\left(V_{2}\right)}\left[e^{-\int_{0}^{t} V_{1}(X(s)) d s}\right] .
$$

The expectation here is taken over all paths starting in $x$ at time 0 and arriving in $x_{t}$ at time $t$ with marginal distribution density

$$
f_{V_{2}}(z ; s)=\frac{d}{d z} F_{V_{2}}(z ; s)=\frac{d}{d z} \operatorname{Prob}\left[X(s) \leq z \mid X(0)=x, X(t)=x_{t}\right]
$$

given by

$$
f_{V_{2}}(z ; s)=\frac{I_{\left[V_{2}(x)\right]}^{\alpha}(x, z, s) I_{\left[V_{2}(x)\right]}^{\alpha}\left(z, x_{t}, t-s\right)}{I_{\left[V_{2}(x)\right]}^{\alpha}\left(x, x_{t}, t\right)} .
$$

Making use of such a decomposition, the functional integral is written as an expectation of a functional involving $V_{1}(x)$. This means that the problem is reduced to the issue of finding an approximation for such an expectation.

\subsection{Upper and lower bound for the expectation}

In order to arrive at a closed-form approximation for the remaining expectation, we will construct an upper and a lower bound, which eventually can be combined to give an accurate approximation.

1. For the upper bound, we make use of the concept of convex ordering, and the relation with comonotonic risks. The basic idea is to exchange the original stochastic variable for a similar variable, for which it is more likely to reach extreme values. In that case, an expectation of a decreasing exponential functional of this new variable will be larger than for the original one. Some definitions and important results about this powerful tool can be found in appendix A.1. For more details, we refer to some earlier contributions, e.g. Dhaene et al. [6,7].

Relying on the notion of convex ordering, it can be shown that

$$
E_{\left(V_{2}\right)}\left[e^{-\int_{0}^{t} V_{1}(X(s)) d s}\right] \leq E_{U}\left[e^{-\int_{0}^{t} F_{V_{1}(X(s))}^{-1}(U) d s}\right]
$$

where $U$ is a $[0,1]$ uniformly distributed variable. The following notations are 
used for the distribution functions:

$$
\begin{gathered}
F_{X(s)}(x)=F_{V_{2}}(x ; s), \quad \text { (cf supra) } \\
F_{V_{1}(X(s))}(x)=\operatorname{Prob}\left[V_{1}(X(s)) \leq x\right]
\end{gathered}
$$

and

$$
F_{V_{1}(X(s))}^{-1}(p)=\inf \left\{x \in \mathbb{R}: F_{V_{1}(X(s))}(x) \geq p\right\},
$$

where $x \in \mathbb{R}$ and $p \in[0,1]$. A proof can be found in appendix A.2.

2. For the lower bound, we suggest to make use of the inequality of Jensen (see e.g. Feynman \& Hibbs [10]), combined with a conditioning on an intermediate time-point. Relying on the inequality of Jensen, it can be shown that

$$
E_{\left(V_{2}\right)}\left[e^{-\int_{0}^{t} V_{1}(X(s)) d s}\right] \geq E_{X(\tau)}\left[e^{-\int_{0}^{\tau} E_{\left(V_{2}\right)}\left[V_{1}(X(s))\right] d s} \cdot e^{-\int_{\tau}^{t} E_{\left(V_{2}\right)}\left[V_{1}(X(s))\right] d s}\right],
$$

where $\tau$ is any point between 0 and $t$. The expectation $E_{X(\tau)}[\ldots]$ means an expectation over all possible values of $X(\tau)$. A proof can be found in appendix A.3.

\section{Barrier option}

A first application of $\delta$-function potential can be found in the pricing of (double) barrier options. The holder of a double knock-out barrier option receives a payoff only if the price of the underlying asset remains in an allowable range $(L, U)$. If $\tau_{[L, U]}=\inf \left\{t \geq 0: X_{t}=L\right.$ or $\left.X_{t}=U\right\}$ is the first hitting time of the stock price process $X$ at the barriers, the payoff of a double knock-out barrier option with maturity date $t>0$ is defined as

$$
h\left(X_{t}\right)=\Phi\left(X_{t}\right) 1_{\left(\tau_{[L, U]}>t\right)}
$$

where $\Phi(x)=(x-K)_{+}$for a call option or $\Phi(x)=(K-x)_{+}$for a put option. From arbitrage-free arguments, the price at time 0 of this financial contract is the expectation under some risk neutral measure $\mathbb{Q}$ of the discounted payoff:

$$
P(x, t)=e^{-r t} E_{x}^{\mathbb{Q}}\left[h\left(X_{t}\right)\right]
$$

where $r$ is the constant risk-free discount factor. If the underlying asset is traded, its risk neutral dynamic follows

$$
d X_{t}=(r-d) X_{t} d t+X_{t} \tilde{\sigma}\left(X_{t}\right) d W_{t}
$$


where $\left\{W_{t}, t \geq 0\right\}$ is a $\mathbb{Q}$-Brownian motion and $d$ is the dividend rate. The function $\tilde{\sigma}(x)$ is called the local volatility. The expectation (24) can also be written as

$$
P(x, t)=e^{-r t} \int_{(L, U)} p_{X}^{A}\left(x, x_{t}, t\right) \Phi\left(x_{t}\right) d x_{t}
$$

where $p_{X}^{A}\left(x, x_{t}, t\right)$ is the transition probability of $X$ absorbed at his first visit at the barriers. We can formulate $p_{X}^{A}\left(x, x_{t}, t\right)$ by means of a Brownian functional integral with infinitely repulsive $\delta$-functions at the location of the barriers. To start with, we transform the process $X$ into a unit volatility process $Y=\phi(X)$ where $\phi(x)=\int^{x} \frac{d z}{\sigma(z)}$ and $\sigma(x)=x \tilde{\sigma}(x)$. The process $Y$ is a diffusion as well, and it is the solution of the stochastic differential equation

$$
d Y_{t}=\mu_{Y}\left(Y_{t}\right) d t+d W_{t}
$$

where

$$
\mu_{Y}(y)=\frac{r-d}{\tilde{\sigma}\left(\phi^{-1}(y)\right)}-\frac{1}{2} \frac{d}{d x} \sigma\left(\phi^{-1}(y)\right)
$$

The transition probability of the process $Y$ satisfies the relation $p_{X}\left(x, x_{t}, t\right)=$ $p_{Y}\left(\phi(x), \phi\left(x_{t}\right), t\right) / \sigma\left(x_{t}\right)$ and is solution of the backward Fokker-Planck equation

$$
-\frac{d}{d t} p\left(y, y_{t}, t\right)=\left[-\frac{1}{2} \frac{d^{2}}{d y^{2}}-\mu_{Y}(y) \frac{d}{d y}\right] p\left(y, y_{t}, t\right) .
$$

The absorbing boundaries have to be imposed on $\phi(L)$ and $\phi(U)$. A trivial application of the results of sections 2 and 3 yields that the following representation of $p_{Y}^{A}\left(y, y_{t}, t\right)$,

$$
\begin{aligned}
p_{Y}^{A}\left(y, y_{t}, t\right)= & \lim _{\gamma \rightarrow+\infty} e^{\int_{y}^{y_{t}} \mu_{Y}(z) d z} \\
& \times \int_{(0, y)}^{\left(t, y_{t}\right)} D y(s) e^{-\frac{1}{2} \int_{0}^{t} \dot{y}^{2}(s) d s-\int_{0}^{t} V(y(s)) d s-\gamma L_{t}^{\phi(L)}(y)-\gamma L_{t}^{\phi(U)}(y)}
\end{aligned}
$$

where $V(y)=\frac{1}{2}\left(\mu_{Y}(y)^{2}+(d / d y) \mu_{Y}(y)\right)$, is the solution of equation (26) subject to the boundary conditions $p_{Y}^{A}\left(\phi(L), y_{t}, t\right)=0$ and $p_{Y}^{A}\left(\phi(U), y_{t}, t\right)=0$. We can directly apply the bounds of section 4 to approximate the transition probability of the diffusion $X$ between the absorbing barriers $L$ and $U$, choosing $V_{1}(y)=V(y)$ and $V_{2}(y)=\gamma \delta(y-\phi(L))+\gamma \delta(y-\phi(U))$ with $\gamma$ going to infinity. The propagator corresponding to the potential $V_{2}(y)$ is the transition probability of a Brownian motion with two absorbing barriers and is known in closed-form, see e.g. Itô \& McKean [15].

We illustrate the method for the local volatility $\tilde{\sigma}(x)=\sigma x^{0.25}, \sigma=0.1$, $r=0.04, d=0, L=80$ and $U=120$. Figure 1 presents the bounds for 
the transition probability of the stock price in the range $(L, U)$ and for the probability of hitting the barriers before the maturity of the contract. Figure 2 demonstrates the accuracy of the bounds for pricing double barrier call options. Table 1 compares the bounds with prices obtained by Monte Carlo simulation. The (asymptotic) 95\% confidence interval given by the Monte Carlo estimate plus or minus 1.96 times the standard error (s.e.) largely overlaps the range between the lower and the upper bound.
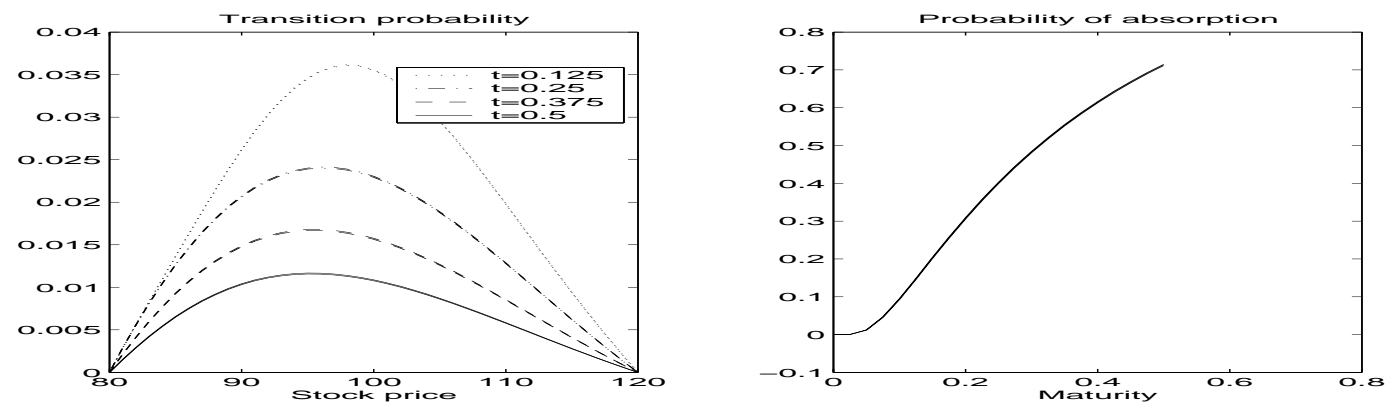

Fig. 1. Bounds for the transition probabilities for different maturity dates $t, L=80$, $U=120, r=0.04, \sigma=0.1, \rho=1.25$ and $X_{0}=100$.

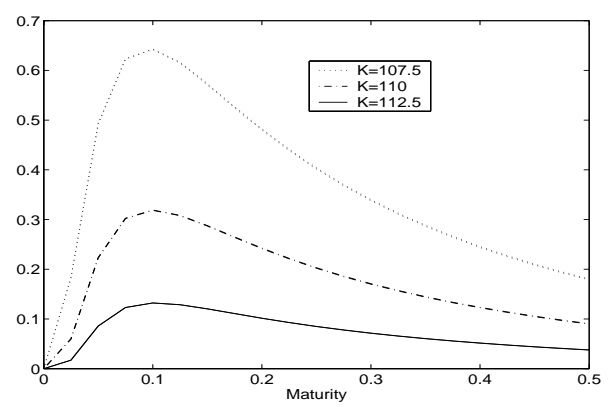

Fig. 2. Bounds for the price of barrier options for several strike prices $K$.

\begin{tabular}{lllllll}
\hline & $\mathrm{t}$ (years) & $\mathrm{MC}$ & s.e & $\mathrm{LB}$ & $\mathrm{UB}$ & r.e. \\
\hline $\mathrm{K}=107.5$ & $t=0.25$ & 0.4088 & 0.0154 & 0.4016 & 0.4037 & 0.005 \\
& $t=0.5$ & 0.1961 & 0.0108 & 0.1929 & 0.1948 & 0.0098 \\
\hline $\mathrm{K}=110$ & $t=0.25$ & 0.2136 & 0.0105 & 0.2021 & 0.2031 & 0.0049 \\
& $t=0.5$ & 0.0973 & 0.0069 & 0.0969 & 0.0978 & 0.0092 \\
\hline $\mathrm{K}=112.5$ & $t=0.25$ & 0.0861 & 0.0062 & 0.0847 & 0.0851 & 0.0047 \\
& $t=0.5$ & 0.0424 & 0.0039 & 0.0406 & 0.0409 & 0.0073 \\
\hline
\end{tabular}

Table 1

Price obtained by Monte Carlo simulation (MC) and the standard error (s.e.) with 10000 paths, the lower bound (LB), the upper bound (UB) and the relative length interval (r.e.) 


\section{Discontinuous local volatility}

An application of skew functional integrals can be found in the pricing of call options when the local volatility is discontinuous. In the Black-Scholes setting, the local volatility function $\tilde{\sigma}(x)$ is assumed to be constant. Nevertheless, empirical evidences suggest that the volatility implied by the price of traded options varies with the maturity and the strike price. The feature that the implied volatility is higher for low strike is referred in the literature as the volatility skew. The term volatility smile relates to implied volatility shapes with a minimum for a particular strike. For further details on the relation between local and implied volatility, we refer e.g. to Derman \& Kani [8]. In this section, we allow for volatility skew and smile through a discontinuous local volatility. In a recent paper, McCauley \& Gunaratne [19] argue in favor of a discontinuous volatility. Eraker et al. [9] provide also empirical evidence that an addition of jumps in volatility significantly improves the explaining power of returns data on the S\&P 500 and Nasdaq 100 index returns.

We assume that the dynamic of the stock under the risk-neutral measure is

$$
d X_{t}=r X_{t} d t+X_{t} \tilde{\sigma}\left(X_{t}\right) d W_{t}
$$

where the local volatility function $\tilde{\sigma}(x)$ is continuous except at the point $x=$ $a>0$ where the difference $\tilde{\sigma}(a+)-\tilde{\sigma}(a-)$ is bounded. An application of the Tanaka formula, see e.g. Revuz \& Yor [24], yields that the transformed process $Y=\phi(X)$ where $\phi(x)=\int^{x} \frac{d z}{\sigma(z)}$ is solution of the following stochastic differential equation

$$
d Y_{t}=\mu_{Y}\left(Y_{t}\right) d t+d W_{t}+\frac{1}{2}\left(\frac{\sigma(a-)-\sigma(a+)}{\sigma(a-) \sigma(a+)}\right) d L_{t}^{a}(X)
$$

where

$$
\mu_{Y}(y)=\frac{r}{\tilde{\sigma}\left(\phi^{-1}(y)\right)}-\frac{1}{2} \frac{d}{d x} \sigma\left(\phi^{-1}(y)\right) .
$$

From the following relation, see e.g. Revuz \& Yor [24],

$$
\begin{aligned}
L_{t}^{\phi(a)}(Y) & =\frac{1}{2}\left(L_{t}^{\phi(a+)}(Y)+L_{t}^{\phi(a-)}(Y)\right) \\
& =\frac{L_{t}^{a+}(X)}{2 \sigma(a+)}+\frac{L_{t}^{a-}(X)}{2 \sigma(a-)} \\
& =\frac{\sigma(a+)+\sigma(a-)}{2 \sigma(a+) \sigma(a-)} L_{t}^{a}(X),
\end{aligned}
$$

we finally obtain that $d Y_{t}=\mu_{Y}\left(Y_{t}\right) d t+d W_{t}+(2 \alpha-1) d L_{t}^{\phi(a)}(Y)$ with $\alpha=$ 
$\sigma(a-) /(\sigma(a-)+\sigma(a+))$. This transformation enables us to formulate the price of a European option on $X$ by means of a skew functional integral. The price of a call option with strike price $K$ can be written as

$$
\begin{aligned}
P(x, t) & =e^{-r t} \int_{K}^{+\infty} p_{X}\left(x, x_{t}, t\right)\left(x_{t}-K\right) d x_{t} \\
& =e^{-r t} \int_{K}^{+\infty} p_{Y}\left(\phi(x), \phi\left(x_{t}\right), t\right)\left(x_{t}-K\right) \frac{d x_{t}}{\sigma\left(x_{t}\right)} .
\end{aligned}
$$

When the domain of the diffusion $Y$ is the whole real line, its transition density can be expressed by means of a skew functional integral. Indeed, the transition density $p_{Y}\left(y, y_{t}, t\right)$ is the solution of the backward Fokker-Planck equation (26), subject to the boundary condition $(1-\alpha) \frac{d}{d y} p\left(\phi(a-), y_{t}, t\right)=\alpha \frac{d}{d y} p\left(\phi(a+), y_{t}, t\right)$, see e.g. Itô \& McKean [15]. Applying the results of sections 2 and 3, tedious but straightforward calculations provide the following representation for $p_{Y}\left(y, y_{t}, t\right)$ :

$$
p_{Y}\left(y, y_{t}, t\right)=e^{\int_{y}^{y_{t}} \mu_{Y}(z) d z} \times \int_{(0, y)}^{\left(t, y_{t}\right)} D y_{\alpha}(s) e^{-\frac{1}{2} \int_{0}^{t} \dot{y}_{\alpha}^{2}(s) d s-\int_{0}^{t} V\left(y_{\alpha}(s)\right) d s-\gamma L_{t}^{\phi(a)}\left(y_{\alpha}\right)}
$$

where $V(y)=\frac{1}{2}\left(\mu_{Y}(y)^{2}+(d / d y) \mu_{Y}(y)\right)$ and

$$
\gamma=\alpha \mu_{Y}(\phi(a+))-(1-\alpha) \mu_{Y}(\phi(a-))
$$

In order to approximate the transition probability of the diffusion $Y$, use can be made of the bounds of section 4, choosing $V_{1}(y)=V(y)$ and $V_{2}(y)=$ $\gamma \delta(y-\phi(a))$. The propagator corresponding to the potential $V_{2}(y)$ is the transition probability of a skew Brownian motion killed when the time spent in the vicinity of $a$ (measured by the local time), exceeds an independent exponential random variable with parameter $\gamma$. The transition function of this non-conservative diffusion is provided in appendix A.4. When $\alpha=1$ or $\alpha=0$, we recover the transition density of the elastic Brownian motion, see e.g. Itô \& McKean [15].

We illustrate the accuracy of the bounds for the discontinuous local volatility function

$$
\tilde{\sigma}(x)= \begin{cases}\sigma_{1} x^{\rho}, & x<a \\ \sigma_{2} x^{\rho}, & x \geq a,\end{cases}
$$

with $a=100, \sigma_{1}=0.2, \sigma_{2}=0.25, \rho=-0.25$. The domain of the diffusion $X$ is the whole real line ${ }^{2}$. This can lead to economical contradiction, however, the probability of reaching negative values is negligible. Figure 3 and 4 present

$\overline{2}$ If the domain of the stochastic process $Y$ is $(0, \infty)$ instead of $(-\infty,+\infty)$, the 
the bounds for the transition probability ${ }^{3}$ and for the price of call options. The transition probability exibits a discontinuity at the level of the barrier. Table 2 compares the bounds with prices obtained by Monte Carlo simulation. The (asymptotic) 95\% confidence interval given by the Monte Carlo estimate plus or minus 1.96 times the standard error (s.e.) largely overlaps the range between the lower and the upper bound.

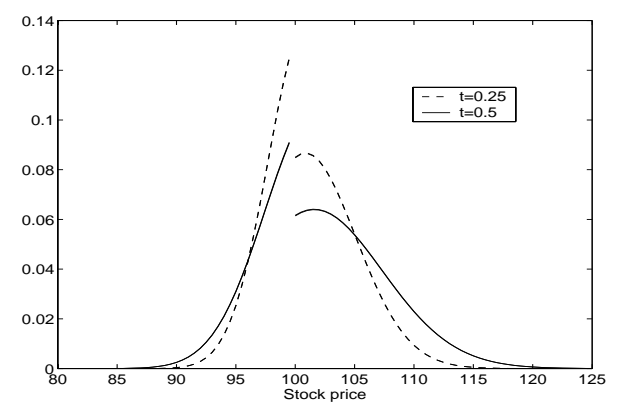

Fig. 3. Bounds for the transition probabilities with different maturity dates $t$, $a=100, r=0.04, \sigma_{1}=0.2, \sigma_{2}=0.25, \rho=0.75$ and $X_{0}=100$.

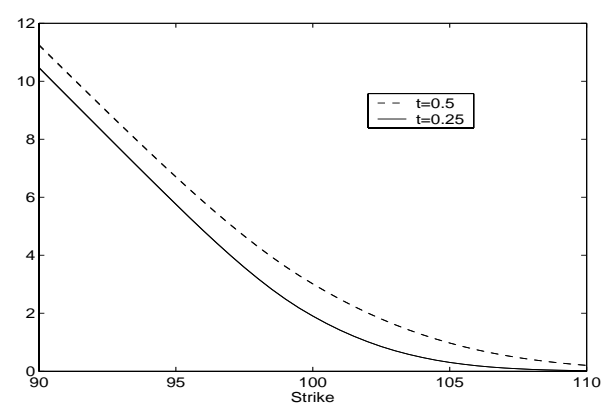

Fig. 4. Bounds for the price of European call option as a function of the strike, $a=100, r=0.04, \sigma_{1}=0.2, \sigma_{2}=0.25, \rho=0.75$ and $X_{0}=100$.

\section{Acknowledgements}

The authors wish to thank the "Onderzoeksraad K.U.Leuven" for a GOA grant. They also thank the referee for his useful comments and helpful suggestions.

differential part $D y_{\alpha}(s) e^{-\frac{1}{2} \int_{0}^{t} \dot{y}_{\alpha}^{2}(s) d s}$ has to be replaced by $D_{+} y_{\alpha}(s) e^{-\frac{1}{2} \int_{0}^{t} \dot{y}_{\alpha}^{2}(s) d s}=$ $\lim _{\gamma \rightarrow+\infty} D y_{\alpha}(s) e^{-\frac{1}{2} \int_{0}^{t} \dot{y}_{\alpha}^{2}(s) d s-\gamma L_{t}^{0}\left(y_{\alpha}\right)}$ for $y, y_{t}>0$.

3 The bounds are no longer transition densities as the total mass is smaller respectively larger than one. To restore this desirable property, we suggest the use of the following convex combination

$$
\tilde{p}_{Y}\left(y, y_{t}, t\right)=z p_{Y}^{\text {low }}\left(y, y_{t}, t\right)+(1-z) p_{Y}^{u p p}\left(y, y_{t}, t\right)
$$

where $z$ is choosen such that $\int \tilde{p}_{Y}\left(y, y_{t}, t\right) d y_{t}=1$. 


\begin{tabular}{lllllll}
\hline & $\mathrm{t}$ (years) & $\mathrm{MC}$ & $\mathrm{s.e}$ & $\mathrm{LB}$ & $\mathrm{UB}$ & r.e. \\
\hline $\mathrm{K}=100$ & $t=0.25$ & 1.8847 & 0.0264 & 1.9035 & 1.9056 & 0.0011 \\
& $t=0.5$ & 3.0243 & 0.0391 & 3.0116 & 3.0162 & 0.0015 \\
\hline $\mathrm{K}=105$ & $t=0.25$ & 0.3167 & 0.0105 & 0.3087 & 0.3098 & $6.4 e^{-4}$ \\
& $t=0.5$ & 0.9646 & 0.0228 & 0.9735 & 0.9746 & 0.0011 \\
\hline $\mathrm{K}=110$ & $t=0.25$ & 0.0190 & 0.0022 & 0.0185 & 0.0185 & $5.4 e^{-4}$ \\
& $t=0.5$ & 0.2015 & 0.0101 & 0.2032 & 0.2034 & $9.8 e^{-4}$ \\
\hline
\end{tabular}

Table 2

Price obtained by Monte Carlo simulation (MC) and the standard error (s.e.) with 10000 paths, the lower bound (LB), the upper bound (UB) and the relative length interval (r.e.)

\section{Appendix}

\section{A.1 About convex ordering}

In this section, we briefly recall the most important definitions and results regarding convex ordering. For proofs and more details, we refer to $[6,7]$.

The notion of convex ordering

A variable $A$ is said to be smaller than $B$ in convex ordering,

$$
A \leq_{c x} B
$$

if for each convex function $u: \mathbb{R} \rightarrow \mathbb{R}: x \mapsto u(x)$, the expected values (provided they exist) are ordered as

$$
E[u(A)] \leq E[u(B)]
$$

Since convex functions take on their largest values in the tails, the variable $B$ is more likely to take on extreme values than the variable $A$, and thus it can be interpreted as more dangerous. For the application in the present contribution, we attain our goal, if we can construct such a more dangerous or convex larger variable for the integral $\int_{0}^{t} V(X(s)) d s$. Making use of comonotonic variables, this can perfectly be done. 


\section{Construction of a convex larger variable}

If a variable $A$ consists of a sum of random variables $X_{1}, X_{2}, \ldots, X_{n}$, then replacing the copula of $\left(X_{1}, X_{2}, \ldots, X_{n}\right)$ by the comonotonic copula, yields a sum that is a convex larger variable than this variable $A$. Or,

$$
\sum_{i=1}^{n} X_{i} \leq_{c x} \sum_{i=1}^{n} F_{X_{i}}^{-1}(U),
$$

where $U$ is an arbitrary random variable, uniformly distributed on $[0,1]$. The notation $F_{X_{i}}$ is used for the distribution function of the variable $X_{i}$, and the inverse distribution is defined in the classical way as

$$
F_{X_{i}}^{-1}(p)=\inf \left\{x \in \mathbb{R}: F_{X_{i}}(x) \geq p\right\} .
$$

Note that the corresponding terms in both sums are all mutually identically distributed. Both sums differ in the interdependence structure; the convex largest sum is the sum with the most dangerous interdependencies. This result can be generalized for a sum of functions of variables as follows:

$$
\sum_{i=1}^{n} g_{i}\left(X_{i}\right) \leq_{c x} \sum_{i=1}^{n} F_{g_{i}\left(X_{i}\right)}^{-1}(U),
$$

where $U$ is an arbitrary random variable, uniformly distributed on $[0,1]$. Finally, when taking limits, the construction is also valid for integrals:

$$
\int_{0}^{t} g(X(s)) d s \leq_{c x} \int_{0}^{t} F_{g(X(s))}^{-1}(U) d s,
$$

where $U$ is an arbitrary random variable, uniformly distributed on $[0,1]$.

\section{A.2 Proof for the upper bound of section 4.1}

Following the result of (35), we know that the variable

$$
A=\int_{0}^{t} V_{1}(X(s)) d s
$$

is smaller in convex ordering than

$$
B=\int_{0}^{t} F_{V_{1}(X(s))}^{-1}(U) d s .
$$

Hence, since the negative exponential function is convex, the result follows immediately from the definition of convex ordering. 


\section{A.3 Proof for the lower bound of section 4.1}

We start by writing

$$
E_{\left(V_{2}\right)}\left[e^{-\int_{0}^{t} V_{1}(X(s)) d s}\right]=E_{\Lambda}\left\{E_{\left(V_{2}\right)}\left[e^{-\int_{0}^{t} V_{1}(X(s)) d s} \mid \Lambda\right]\right\}
$$

for an arbitrary variable $\Lambda$. For a choice of $\Lambda \equiv X(\tau)$ with $\tau$ between 0 and $t$, the inequality of Jensen immediately leads to the lower bound of (22).

\section{A.4 Exact result from $\delta$-function perturbation}

For the skew Brownian motion with $V(x)=0$, it follows that

$$
\begin{aligned}
G_{(0)}^{\alpha, \gamma}\left(x, x_{t}, s\right) & =\int_{0}^{\infty} e^{-s t} d t \int_{(0, x)}^{\left(t, x_{t}\right)} D x_{\alpha}(s) e^{-\frac{1}{2} \int_{0}^{t} \dot{x}_{\alpha}^{2}(s) d s-\gamma L_{t}^{a}\left(x_{\alpha}\right)} \\
& =\int_{0}^{\infty} e^{-s t} d t \int_{(0, x)}^{\left(t, x_{t}\right)} D x_{\alpha}(s) e^{-\frac{1}{2} \int_{0}^{t} \dot{x}_{\alpha}^{2}(s) d s-\frac{\gamma}{2 \alpha} L_{t}^{a+}\left(x_{\alpha}\right)} \\
& =G_{(0)}^{\alpha}\left(x, x_{t}, s\right)-\frac{G_{(0)}^{\alpha}(x, a+, s) G_{(0)}^{\alpha}\left(a+, x_{t}, s\right)}{G_{(0)}^{\alpha}(a+, a+, s)+2 \alpha / \gamma} .
\end{aligned}
$$

Inserting the expression for $G_{(0)}^{\alpha}\left(x, x_{t}, s\right)$, we obtain

$$
G_{(0)}^{\alpha, \gamma}\left(x, x_{t}, s\right)= \begin{cases}G_{(0)}^{\alpha}\left(x, x_{t}, s\right)-2 \alpha \frac{\gamma e^{-\sqrt{2 s}\left(\left|x_{t}-a\right|+|a-x|\right)}}{\sqrt{2 s}(\sqrt{2 s}+\gamma)}, & x_{t} \geq a \\ G_{(0)}^{\alpha}\left(x, x_{t}, s\right)-2(1-\alpha) \frac{\gamma e^{-\sqrt{2 s}\left(\left|x_{t}-a\right|+|a-x|\right)}}{\sqrt{2 s}(\sqrt{2 s}+\gamma)}, & x_{t}<a .\end{cases}
$$

The inversion of the Laplace transform, see e.g. Abramowitz \& Stegun [1], provides the following expression

$$
\begin{aligned}
& I_{(0)}^{\alpha, \gamma}\left(x, x_{t}, t\right) \\
& = \begin{cases}I_{(0)}^{\alpha}\left(x, x_{t}, t\right)-\alpha \gamma e^{\gamma k} e^{\frac{1}{2} \gamma^{2} t} \operatorname{erfc}\left(\gamma \sqrt{t / 2}+\frac{k}{\sqrt{2 t}}\right), & x_{t} \geq a \\
I_{(0)}^{\alpha}\left(x, x_{t}, t\right)-(1-\alpha) \gamma e^{\gamma k} e^{\frac{1}{2} \gamma^{2} t} \operatorname{erfc}\left(\gamma \sqrt{t / 2}+\frac{k}{\sqrt{2 t}}\right), & x_{t}<a,\end{cases}
\end{aligned}
$$

with $k=\left(\left|x_{t}-a\right|+|a-x|\right)$. 


\section{References}

[1] Abramowitz M., \& Stegun I.A. (1970). Handbook of mathematical functions, Dover, $1046 \mathrm{p}$.

[2] Baaquie B.E. (1997). "A path integral approach to option pricing with stochastic volatility: some exact results", Journal de Physique I, vol.7(12), p.1733-1753.

[3] Cantrell R. S. \& Cosner C. (1999) "Diffusion models for population dynamics incorporating individual behavior at boundaries: applications to refuge design", Theoretical Population Biology, Vol. 55, Issue 2, p.189-207.

[4] Carreau M. (1992) "The functional integral for a free particle on a half-plane", Journal of Mathematical Physics, Vol. 33(12), p.4139-4147.

[5] Chiarella C., El-Hassan N., \& Kucera A. (1999). "Evaluation of Americal option prices in a path integral framework using Fourier-Hermite series expansions", Journal of Economic Dynamics and Control, vol.23, p.1387-1424.

[6] Dhaene J., Denuit M., Goovaerts M.J., Kaas R. \& Vyncke D. (2002). "The concept of comonotonicity in actuarial science and finance: theory", Insurance: Mathematics and Economics, vol.31(1), p.3-34.

[7] Dhaene J., Denuit M., Goovaerts M.J., Kaas R. \& Vyncke D. (2002). "The concept of comonotonicity in actuarial science and finance: applications", Insurance: Mathematics and Economics, vol.31(2), p.133-162.

[8] Derman E. and Kani I. (1996) "The local volatility surface: unlocking the infomartion in index option prices, Financial Analysts Journal, vol. 53, p.25-36.

[9] Eraker B., Johannes M.S. \& Polson N. (2003). "The impact of jumps in volatility and returns", Journal of Finance, vol.58(3), p.1269-1300.

[10] Feynman R.P., \& Hibbs A.R. (1965). Quantum mechanics and path integrals, McGraw-Hill Book Company, 365 p.

[11] Goovaerts M., De Schepper A., \& Decamps M. (2003). "Closed-form approximations for diffusion densities: a path integral approach", Journal of Applied and Computational Mathematics, vol.165, p.337-364.

[12] Goovaerts M., Babcenco A., \& Devreese J. (1973). "A new expansion method in the Feynman path integral formalism: application to the one-dimensional deltafunction potential", Journal of Mathematical Physics, vol.14(5), p.554-559.

[13] Grosche C. (1990). "path integrals for potential problems with $\delta$-function perturbation", Journal of Physics A, vol.23, p.5205-5234.

[14] Grosche C. (1993). " $\delta$-function perturbations and boundary problems by path integration", Annalen der Physik, vol.2(6), p.557-589.

[15] Itô K., \& McKean H.P. (1965). Diffusion processes and their sample paths, Academic Press, New York, 321 p. 
[16] Kleinert H. (2002). "Option pricing from path integral for non-Gaussian fluctuations. Natural martingale and application to truncated Levy distributions", Physica A, vol.312(1), p.217-242.

[17] Legall J.F. (1982). "Temps locaux et équations différentielles stochastiques", Lecture Notes in Mathematics, Springer-Verlag, 986 p.

[18] Matacz A. (2002). "Path dependent option pricing: the path integral partial averaging method", Journal of Computational Finance, vol.6(2).

[19] McCauley J.L., Gunaratne G.H. (2003). " An empirical model of volatility returns and option pricing", Physica A, vol.329, p.178-198.

[20] Ming Zhang (2000). " Calculation of Diffusive Shock Acceleration of Charged Particles by Skew Brownian Motion", The Astrophysical Journal, 541, 428-435.

[21] Montagna G., Moreni N., \& Nicrosini O. (2002). "A path integral way to option pricing", Physica A, vol.310(3), p.450-466.

[22] Montagna G., \& Nicrosini O. (2002). "Efficient option pricing with path integral", European Physical Journal B, vol.27(2), p.249-256.

[23] Montagna G., Morelli M., Nicrosini O., Amato P., \& Farina M. (2003). "Pricing derivatives by path integral and neural networks", Physica A, vol.324(1), p.189195.

[24] Revuz D., \& Yor M. (1991). Continuous martingales and Brownian motion, Springer-Verlag, Berlin, 533 p.

[25] Rosa-Clot M., \& Taddei S. (2002). "A path integral approach to derivative security pricing", International Journal of Theoretical and Applied Finance, vol.5(2), p.123-146. 